Correo: innova@uide.edu.ec

\title{
Formación de docentes en el uso y apropiación de las TIC
}

\section{Teacher training in the use and ownership of TIC}

Jorge Enrique Díaz Pinzón

Institución Educativa General Santander, Colombia

Autor para correspondencia: jorgediaz333@gmail.com

Fecha de recepción: 11 de Mayo de 2017 - Fecha de aceptación: 15 de Septiembre de 2017

\section{Resumen \\ Para establecer el uso y apropiación de las TIC en las competencias del docente de primaria, básica y media, en el año 2016 se aplicó una encuesta de reactivos cerrados a una muestra de 100 docentes del municipio de Soacha-Cundinamarca, Colombia. Se analizaron 6 preguntas de la encuesta relacionadas con un taller de formación. Se trabajaron seis hipótesis de investigación, y la información se procesó con el paquete estadístico SPSS v.23, con una prueba de Chi Cuadrado para las hipótesis. Al calcular el Coeficiente de Contingencia $\mathrm{C}$ de 0,087 concluimos que esta es una relación baja, se demostró que no hay una relación entre el sexo de los docentes y los contenidos vistos del portal Colombia aprende, laboratorios PHET, banco de recursos, motivación para implementar recursos digitales tenencia y uso apropiado de tabletas.}

Palabras clave: competencias del docente; formación de docentes; tecnologías de la información

\begin{abstract}
In order to establish the use and appropriation of ICT in primary, secondary and elementary teachers' competences, in 2016, a closed reagent survey was applied to a sample of 100 teachers from the municipality of Soacha-Cundinamarca, Colombia. Six survey questions related to a training workshop were analyzed. Six research hypotheses were worked out, and the information was processed with the SPSS v.23 statistical package, with a Chi-square test for the hypotheses. When calculating the Contingency Coefficient $\mathrm{C}$ of 0.087 , we conclude that this is a low relation, it was shown that there is no relationship between the sex of the teachers and the contents seen on the portal Colombia learns, PHET laboratories, resource bank, motivation to implement resources Digital holding and proper use of tablets.
\end{abstract}

Key words: teacher competences; teacher training; information technology 


\section{Introducción}

La utilización de objetos virtuales de aprendizaje (OVA) en el proceso de enseñanzaaprendizaje en la educación escolar ha venido incrementándose a nivel mundial por su contribución en el mejoramiento de la comunicación entre estudiantes y docentes, (Zapata, 2003) citado por (Espinosa, Restrepo y Aránzazu, 2016) y en el diseño de actividades prácticas de aprendizaje y de evaluación, como lo desarrollado por (Díaz, 2016), cuando demostró que, el rendimiento académico de los estudiantes de grado octavo mejoró significativamente al utilizar el simulador PhET en la enseñanza de las fracciones equivalentes. El desarrollo de las nuevas tecnologías de la información y la comunicación relacionadas con el internet, ha abierto nuevos espacios en el ámbito educativo. El diseño de sitios web que integran múltiples aplicaciones multimedia, está dotando a los docentes en general, de nuevos ambientes de aprendizaje donde predomina la interactividad y el rol del profesor innovador, facilitando al estudiante la construcción de su propio conocimiento.

Por lo anterior, se implementó a partir del año 2016, en la secretaría de educación de Soacha-Cundinamarca una red de formador de formadores del CIER centro del Ministerio de Educación Nacional, con el objetivo de incorporar una red educativa digital en los colegios oficiales del municipio de Soacha, además de tener la finalidad de iniciar un proceso de innovación en el municipio. Un claro ejemplo de esto se presenta en el Marco de Acción de Dakar Educación para todos realizado por la UNESCO en el 2000, en donde los países de América Latina se comprometieron a:

Adoptar y fortalecer, donde estén ya en uso, las TIC para mejorar la toma de decisiones de política y planificación de los sistemas educativos; la administración de las escuelas, facilitando los procesos de descentralización y autonomía de la gestión; capacitar a los administradores y maestros en la introducción y manejo de las TIC (UNESCO, 2000, p.41).

\section{Metodología}

Para llevar a cabo el proceso investigativo se diseñó una encuesta de reactivos cerrados con seis preguntas donde el docente contestaba Si o NO a la pregunta, teniendo en cuenta variables de tipo cualitativo, fue aplicada en el mes de septiembre de 2016 a los docentes de la Secretaría de Educación de Soacha-Cundinamarca, Colombia.

La técnica empleada fue muestreo simple, utilizando una distribución uniforme en la elección de los encuestados. Para una población total de 100 docentes de instituciones educativas oficiales.

\section{Muestra.}

La muestra está representada por docentes de las instituciones oficiales del municipio de Soacha-Cundinamarca

\section{Fórmula para calcular el tamaño de la muestra}

Se utilizó la siguiente fórmula para calcular el tamaño de la muestra: 


$$
n=\frac{Z^{2} \sigma^{2} N}{e^{2}(N-1)+Z^{2} \sigma^{2}}
$$

Dónde:

$\mathrm{n}=$ el tamaño de la muestra.

$\mathrm{N}$ = tamaño de la población.

$\sigma=$ Desviación estándar de la población que, generalmente cuando no se tiene su valor, suele utilizarse un valor constante de 0,5 .

$\mathrm{Z}=$ Valor obtenido mediante niveles de confianza. Es un valor constante que, si no se tiene su valor, se lo toma en relación al 95\% de confianza equivale a 1,96 (como más usual) o en relación al $99 \%$ de confianza equivale 2,58 , valor que queda a criterio del investigador.

e = Límite aceptable de error muestral que, generalmente cuando no se tiene su valor, suele utilizarse un valor que varía entre el $1 \%(0,01)$ y $9 \%(0,09)$, valor que queda a criterio del encuestador.

Se tiene $N=120$, para el $99 \%$ de confianza $Z=2,58$, y como no se tiene los demás valores se tomará $^{\sigma}=0,5 \mathrm{y} \mathrm{e}=0,05$.

Reemplazando los valores en la fórmula se obtiene:

$$
n=\frac{120(0,5)^{2}(2,58)^{2}}{(120-1)(0,05)^{2}+(0,5)^{2}(2,58)^{2}}
$$

La encuesta fue aplicada con la participación voluntaria de los docentes. La base de datos se elaboró en una hoja electrónica en Google Docs. De acuerdo con el formato delineado en la encuesta y se realizó un estricto control en el proceso investigativo, con el fin de garantizar los resultados y conclusiones creadas a la veracidad de la información.

\section{Resultados}

A fin de establecer una viable relación entre el uso y formación de las Tecnologías de Información y la Comunicación (TIC) y el sexo de los docentes encuestados, se llevó a cabo un análisis correlacional entre las variables que aparecen en las 6 preguntas de la encuesta. Para el análisis estadístico de los datos, se utilizó el paquete estadístico SPSS versión 23.0.

Para calcular la correlación entre variables se utilizaron dos pruebas estadísticas no paramétricas, que "representan pruebas de hipótesis en las que se tienen datos de nivel nominal u ordinal, y que están libres de supuestos acerca de la forma de la población" (Lind, 
2000:448). Estas pruebas son las denominadas Chi Cuadrado de Pearson, y el Coeficiente de Contingencia $\mathrm{C}$.

La prueba Chi Cuadrada "consiste en el análisis de la diferencia entre las expectativas con base en la distribución planteada como hipótesis y los datos reales que aparecen en la muestra” (Webster, 2000:466).La prueba $\mathrm{X}^{2}$ consiente establecer si dos variables cualitativas están o no asociadas, preliminarmente fijado, que ambas son independientes. Para su cómputo es necesario calcular las frecuencias esperadas (aquellas que deberían haberse observado si la hipótesis de independencia fuese cierta), y compararlas con las frecuencias observadas en la realidad.

De otro modo, la segunda prueba no paramétrica utilizada es el Coeficiente de Contingencia $\mathrm{C}$, que se define como: $\mathrm{C}=$

$$
\sqrt{\frac{\chi^{2}}{N^{2}}}
$$

Donde $\mathrm{X}^{2}$ es el valor calculado de la prueba de Ji-Cuadrado y $n$ es el número de datos. El valor de $\mathrm{C}$ varía entre 0 y $1 . \mathrm{C}=0$, significa que no hay asociación entre las variables. $\mathrm{C}$ $>0.30$, indica una buena asociación entre las variables. (Acuña, 2005:14)

Un Coeficiente de Contingencia $\mathrm{C}$ mayor a 0.3 y menor a 0.4 considera la relación entre las variables buena, mientras que un C $>0.40$ establece una relación alta, un C entre 0.25 y 0.30 es una relación moderada y $\mathrm{C}<0.25$ la tomamos como una relación baja.

Tomando como referencia las pruebas antes señaladas, en este apartado se evalúa el grado o nivel de relación o no relación entre las principales variables que caracterizan a las Tecnologías de la Información y la Comunicación (TIC) y las diversas características de los docentes encuestados.

A continuación se presentan los resultados de las pruebas estadísticas realizadas:

Estudio de las diferencias entre el sexo de los docentes en el uso y la formación TIC (El portal Colombia aprende, laboratorios PHET, banco de recursos, tenencia y uso apropiado de tabletas y motivación para implementar recursos digitales).

El portal Colombia aprende, ayuda a los docentes en su práctica pedagógica.

No se calculó ningún estadístico porque El portal Colombia aprende, ayuda a los docentes en su práctica pedagógica es una constante, es decir el 99\% de los docentes contesto SI a la pregunta.

Cree usted que los laboratorios PHET, son una herramienta didáctica novedosa e innovadora, que facilita el aprendizaje en los estudiantes. 
No se calculó ningún estadístico porque laboratorios Phet, facilita el aprendizaje en los estudiantes es una constante, es decir el $99 \%$ de los docentes contesto SI a la pregunta. Las hipótesis contrastadas son las siguientes:

\section{Del banco de recursos, podría emplearlos en proyectos con los estudiantes.}

Ho: No existe relación entre el sexo de los docentes y la posibilidad de emplear en banco de recursos en proyectos con los estudiantes.

Ha: Si existe relación entre el sexo de los docentes y la posibilidad de emplear en banco de recursos en proyectos con los estudiantes.

La Chi Cuadrado calculada es de 0,008 Al ser este valor mayor a la Chi Cuadrada tabulada, al $95 \%$ de confianza y 1 grados de libertad y grado de significancia $(0,927)$, este es mayor que el $\alpha=0,05$ establecido; por lo tanto, aceptamos la Hipótesis Nula (Ho) de que No existe relación entre el sexo de los docentes y la posibilidad de emplear en banco de recursos en proyectos con los estudiantes. Al calcular el Coeficiente de Contingencia $\mathrm{C}$ de 0.009 concluimos que esta es una relación baja.

En su institución educativa tienen las tabletas donadas por el Ministerio.

Ho: No existe relación entre el sexo de los docentes y la tenencia de tabletas donadas por el ministerio.

Ha: Si existe relación entre el sexo de los docentes y la tenencia de tabletas donadas por el ministerio.

La Chi Cuadrado calculada es de 0,005 Al ser este valor mayor a la Chi Cuadrada tabulada, al $95 \%$ de confianza y 1 grados de libertad y grado de significancia $(0,946)$, este es mayor que el $\alpha=0,05$ establecido; por lo tanto, aceptamos la Hipótesis Nula (Ho) de que No existe relación entre el sexo de los docentes y la de tabletas donadas por el ministerio. Al calcular el Coeficiente de Contingencia $\mathrm{C}$ de 0.007 concluimos que esta es una relación baja.

Uso adecuado de las tabletas en sus instituciones educativas.

Ho: No existe relación entre el sexo de los docentes y la tenencia de y el uso adecuado de las tabletas en sus instituciones educativas.

Ha: Si existe relación entre el sexo de los docentes y el uso adecuado de las tabletas en sus instituciones educativas.

La Chi Cuadrado calculada es de 0,278. Al ser este valor mayor a la Chi Cuadrada tabulada, al $95 \%$ de confianza y 1 grado de libertad y grado de significancia $(0,598)$, este es mayor que el $\alpha=0,05$ establecido; por lo tanto, aceptamos la Hipótesis Nula (Ho) de que No 
existe relación entre el sexo de los docentes y el uso adecuado de las tabletas en sus instituciones educativas.

Al calcular el Coeficiente de Contingencia $\mathrm{C}$ de 0.53 concluimos que esta es una relación alta.

\section{Esta usted motivado para implementar recursos digitales en su práctica pedagógica.}

Ho: No existe relación entre el sexo de los docentes y si motivado para implementar recursos digitales en su práctica pedagógica.

Ha: Si existe relación entre el sexo de los docentes y si motivado para implementar recursos digitales en su práctica pedagógica.

La Chi Cuadrado calculada es de 0,755. Al ser este valor mayor a la Chi Cuadrada tabulada, al $95 \%$ de confianza y 1 grados de libertad y grado de significancia $(0,385)$, este es mayor que el $\alpha=0,05$ establecido; por lo tanto, aceptamos la Hipótesis Nula (Ha) de que No existe relación entre el sexo de los docentes y si está motivado para implementar recursos digitales en su práctica pedagógica. Al calcular el Coeficiente de Contingencia C de 0,087 concluimos que esta es una relación baja.

Tabla 1.Valores de Chi Cuadrado y Coeficiente de Contingencia C Relacionadas con el Género de los Docentes y algunas Variables Relacionadas con la Formación, y el Uso de las TIC

\begin{tabular}{lcccc}
\hline tributo & $\begin{array}{c}\text { Chi } \\
\text { Cuadrada } \\
\text { (Calculada) }\end{array}$ & $\begin{array}{c}\text { Grados } \\
\text { de Libertad }\end{array}$ & $\begin{array}{c}\text { Sig. } \\
\text { Asintótica } \\
\text { (bilateral) }\end{array}$ & $\begin{array}{c}\text { Coeficiente de } \\
\text { Contingencia C }\end{array}$ \\
\hline $\begin{array}{c}\text { 1. El portal Colombia } \\
\text { aprende, ayuda a los docentes } \\
\text { en su práctica pedagógica. }\end{array}$ & $\begin{array}{c}\text { No } \\
\text { calculada }\end{array}$ & & & \\
$\begin{array}{c}\text { 2.Laboratorios PHET, } \\
\text { facilita el aprendizaje en los }\end{array}$ & $\begin{array}{c}\text { No } \\
\text { estudiantes }\end{array}$ & & & \\
$\quad \begin{array}{l}\text { 3.Del banco de recursos } \\
\text { podría emplearlos en }\end{array}$ & 0,008 & 1 & 0,927 & 0.009 \\
proyectos con sus estudiantes & & & & \\
$\quad$ 4. Hay tabletas en su IE & 0,005 & 1 & 0,946 & 0.007 \\
$\quad \begin{array}{l}\text { 5. Uso adecuado de tabletas } \\
\text { 6. Motivación para }\end{array}$ & 0,278 & 1 & 0,598 & 0.53 \\
implementar recursos digitales & 0,755 & 1 & 0,385 & 0.087 \\
\hline
\end{tabular}

\section{Discusión}

Para el caso de los talleres de formación pedagógica en el uso de las TIC, la participación de los docentes de Soacha ha sido alta, en concordancia con el mejoramiento continuo debe considerarse como una necesidad en el proceso educativo; en contraste con otros estudios (Peeraer y Petegem 2011; Umoru 2012), que registran que uno de los factores que afecta la confianza de los docentes en el uso de las TIC es la falta de auto entrenamiento, tanto en destrezas tecnológicas como pedagógicas, aspectos que deben ajustarse para lograr un uso efectivo de las tecnologías en el aprendizaje. 
Según (Espinosa, Restrepo y Aránzazu 2016) "la formación docente por lo tanto, no debe limitarse a talleres y entrenamiento sino también al acompañamiento permanente al docente para la identificación de herramientas acordes con sus métodos de enseñanza", de acuerdo con otros estudios realizados (Khan 2012), los docentes requieren demostraciones sobre el impacto de las TIC para hacer sus enseñanzas más interesantes, motivantes y entretenidas y plantean también la necesidad de crear espacios para la edificación de redes de colaboración que aprueben como lo plantean otros autores (Ryymin, Palonen y Hakkarainen 2008) el intercambio de saberes y el soporte para el uso pedagógico de las TIC.

Teniendo en cuenta que la Secretaría de educación de Soacha, tiene una oferta de formación pedagógica para los docentes en el uso de las TIC en la enseñanza y que la gran parte de los docentes estarían dispuestos a incluir el desarrollo de talleres en su plan de aula, se requiere la ejecución de un proceso de formación y acompañamiento permanente para la identificación de herramientas acordes con sus necesidades de enseñanza, que los estimule a utilizar las TIC en la docencia por medio de la implementación de los conocimientos obtenidos en sus actividades de enseñanza, esto en consonancia con (Espinosa, Restrepo y Aránzazu 2016) donde mencionan "que se requiere mejorar las habilidades informáticas de los docentes de manera que puedan orientar a los estudiantes en el uso de las TIC para el aprendizaje, por medio del desarrollo de competencias digitales".

\section{Conclusiones}

El análisis correlacional, en relación al uso y apropiación de los docentes a las TIC, evidenció que no existe relación entre el sexo de los docentes y las respuestas al banco de recursos, tenencia y uso apropiado de tabletas y motivación para implementar recursos digitales. Además se demostró una relación baja entre el sexo de los docentes y banco de recursos, tenencia y motivación para implementar recursos digitales y una relación alta al uso adecuado de tabletas.

Teniendo en cuenta que la Secretaría de Educación de Soacha tiene proyectada la formación y uso de las tic para el año 2017 en la enseñanza por parte de los docentes estarían dispuestos a incluir el desarrollo de cursos en su plan de trabajo, se requiere la implementación de un proceso de formación y seguimiento permanente en las instituciones para la identificación de herramientas acordes con sus métodos de enseñanza, que los incentive a utilizar las TIC en la docencia por medio de la implementación de los conocimientos adquiridos a sus actividades de aula.

Por otro lado, se requiere mejorar las habilidades informáticas de los docentes de manera que puedan orientar a los estudiantes en el uso de las TIC para el aprendizaje, por medio del desarrollo de competencias digitales.

\section{Bibliografía}

Acuña, E. (2004). Análisis de Datos Categóricos. Departamento de Matemáticas Universidad de Puerto Rico- Mayagüez 
Díaz.J (2016). Aplicación PhET: estrategia de enseñanza aprendizaje de fracciones equivalentes. Revista Criterios, 23(1)111-123.Recuperado el 9 de mayo de 2017 de: http://www.umariana.edu.co/ojs-editorial/index.php/criterios/article/view/1193/1136

Espinosa, H., Restrepo L y Aránzazu D. (2016). Desarrollo de habilidades digitales docentes para implementar ambientes virtuales de aprendizaje en la docencia universitaria. Revista Sophia. Vol. 12, Núm. 2 (2016)

Khan, S. H. (2012). "Integration of ICT Component in Teacher Educational Institutions: An Unavoidable Step towards Transforming the Quality of Present Teacher Education System.” Indian Streams Research Journal 1(5).

Lind, D. Marchal W y Mason R. (2015).Estadística para administración y economía. Alfaomega. 11 Edición.Colombia.

Peeraer, J. y Van Petegem, P (2011). "How to Address Integration of ICT in Teaching Practice? Research on Factors Influencing the Use of ICT in Education.": Education in a technological world: communicating current and emerging research and technological efforts.

Ryymin, E., Palonen T y Hakkarainen K. (2008)."Networking Relations of Using ICT within a Teacher Community.” Computers \& Education, 51(3), 1264-1282. 51: 1264-82.

Umoru, T.A. (2012). "Barriers To The Use Of Information And Communication Technologies In Teaching And Learning Business Education." American Journal of Business Education (Online) 5(5): 575-80.

UNESCO (2000).Foro Mundial sobre la Educación, Dakar 2000.Paris: UNESCO.

Webster, A. (2001).Estadística aplicada a los negocios y la economía. Tercera Edición.McGrawHill 